Ferrata Storti Foundation

\title{
Clinical significance of chromatin-spliceosome acute myeloid leukemia: a report from the Northern Italy Leukemia Group (NILG) randomized trial 02/06
}

Haematologica 2021

Volume 106(10):2578-2587

\section{Correspondence:}

ALESSANDRO RAMBALDI

arambaldi@asst-pg23.it

Received: March 22, 2020.

Accepted: August 25, 2020.

Pre-published: August 27, 2020.

https://doi.org/10.3324/haematol.2020.252825

(C)2021 Ferrata Storti Foundation

Material published in Haematologica is covered by copyright. All rights are reserved to the Ferrata Storti Foundation. Use of published material is allowed under the following terms and conditions:

https://creativecommons.org/licenses/by-nc/4.0/legalcode. Copies of published material are allowed for personal or internal use. Sharing published material for non-commercial purposes is subject to the following conditions:

https://creativecommons.org/licenses/by-nc/4.0/legalcode, sect. 3. Reproducing and sharing published material for commercial purposes is not allowed without permission in writing from the publisher.
Chiara Caprioli, ${ }^{1 *}$ Federico Lussana,${ }^{1 *}$ Silvia Salmoiraghi, ${ }^{1,2}$ Roberta Cavagna, ${ }^{1}$ Ksenija Buklijas, ${ }^{1}$ Lara Elidi, ${ }^{1}$ Pamela Zanghì, ${ }^{1}$ Anna Michelato, ${ }^{1}$ Federica Delaini, ${ }^{1}$ Elena Oldani, ${ }^{1}$ Tamara Intermesoli, ${ }^{1}$ Anna Grassi, ${ }^{1}$ Giacomo Gianfaldoni, ${ }^{3}$ Francesco Mannelli, ${ }^{3}$ Dario Ferrero, ${ }^{4}$ Ernesta Audisio, ${ }^{4}$ Elisabetta Terruzzi, ${ }^{5}$ Lorella De Paoli, ${ }^{6}$ Chiara Cattaneo, ${ }^{7}$ Erika Borlenghi, ${ }^{7}$ Irene Cavattoni, ${ }^{8}$ Monica Tajana, ${ }^{9}$ Anna Maria Scattolin, ${ }^{10}$ Daniele Mattei,,${ }^{11}$ Paolo Corradini, ${ }^{12,13}$ Leonardo Campiotti, ${ }^{14}$ Fabio Ciceri, ${ }^{15}$ Massimo Bernardi, ${ }^{15}$ Elisabetta Todisco ${ }^{16}$ Agostino Cortelezzi ${ }^{17}$ Brunangelo Falini ${ }^{18}$ Chiara Pavoni, ${ }^{1}$ Renato Bassan, ${ }^{10}$ Orietta Spinelli ${ }^{1}$ and Alessandro Rambaldi ${ }^{1,13}$

${ }^{1}$ Azienda Socio-Sanitaria Territoriale (ASST) Ospedale Papa Giovanni XXIII, Bergamo; ${ }^{2}$ FROM Research Foundation, Ospedale Papa Giovanni XXIII, Bergamo; ${ }^{3}$ Azienda Ospedaliera Universitaria Careggi (AOU), Firenze; ${ }^{4} \mathrm{AOU}$ Città della Salute e della Scienza, Torino; ${ }^{5}$ Azienda Ospedaliera San Gerardo, Monza; ${ }^{6}$ Azienda Ospedaliera SS. Antonio e Biagio e Cesare Arrigo, Alessandria; ${ }^{7}$ Spedali Civili, Brescia; ${ }^{8}$ Ospedale San Maurizio, Bolzano; ${ }^{A}$ ASST Ospedale di Cremona, Cremona; ${ }^{10}$ Ospedale dell'Angelo e SS. Giovanni e Paolo, Venezia Mestre; ${ }^{11}$ Azienda Ospedaliera S. Croce e Carle, Cuneo; ${ }^{12}$ Fondazione Istituto di Ricovero e Cura a Carattere Scientifico (IRCCS) Istituto Nazionale dei Tumori, Milano; ${ }^{13}$ Department of Oncology and Hematology, Università degli Studi di Milano, Milano; ${ }^{14}$ Università dell'Insubria, Varese; ${ }^{15}$ IRCCS Ospedale San Raffaele, Milano; ${ }^{16}$ IRCCS Istituto Clinico Humanitas di Rozzano, Rozzano; ${ }^{17}$ Fondazione IRCCS Ca' Granda Ospedale Maggiore Policlinico, Milano and ${ }^{18}$ Università di Perugia, Perugia, Italy

${ }^{*} \mathrm{CC}$ and $\mathrm{FL}$ contributed equally as co-first authors.

\section{ABSTRACT}

S econdary acute myeloid leukemia (sAML) after myelodysplastic or myeloproliferative disorders is a high-risk category currently identified by the clinical history or specific morphological and cytogenetic abnormalities. However, in the absence of these features, uncertainties to identify the secondary nature of some cases, otherwise defined as de novo AML, remain. In order to test whether a chromatinspliceosome (CS) mutational signature might better define the de novo AML group, we analyzed a prospective cohort of 413 newly diagnosed AML patients who were enrolled in a randomized clinical trial (NILG AML 02/06) and who provided samples for accurate cytogenetic and molecular characterization. Among clinically defined de novo AML, $17.6 \%$ carried CS mutations (CS-AML) and showed clinical characteristics closer to sAML (older age, lower white blood cell counts and higher rate of multilineage dysplasia). Outcomes in this group were adverse, more similar to those of sAML as compared to de novo AML (overall survival, $30 \%$ in CS-AML and $17 \%$ in sAML vs. $61 \%$ in de novo AML, $P<0.0001$; disease-free survival, $26 \%$ in CS-AML and $22 \%$ in sAML vs. $54 \%$ of de novo AML, $P<0.001)$ and independently confirmed by multivariable analysis. Allogeneic transplant in first complete remission improved survival in both sAML and CS-AML patients. In conclusion, these findings highlight the clinical significance of identifying CS-AML for improved prognostic prediction and potential therapeutic implications. (NILG AML 02/06; clinicaltrials gov. Identifier: NCT00495287). 


\section{Introduction}

According to the current World Health Organization (WHO) classification, ${ }^{1}$ secondary acute myeloid leukemia (sAML) is defined either by a previous clinical history of hematological disease, the morphological detection of multilineage dysplasia or specific cytogenetic characteristics; the two latter criteria are additive to the clinical history or may be themselves sufficient to diagnose sAML, even in the absence of a known antecedent myelodysplastic syndrome (MDS) or myeloproliferative neoplasm (MPN). ${ }^{1,2}$ However, in clinical practice some uncertainty remains regarding the correct classification of potentially high-risk sAML cases, especially when an antecedent history is not thoroughly documented or does not fully satisfy the diagnostic criteria of MDS. Moreover, morphological examination to assess blast counts and multilineage dysplasia shows inter-observer variability that may impair diagnostic reproducibility. ${ }^{3}$ Finally, cytogenetic analysis, which usually takes a long turnaround time of 510 days, may not be always informative because of technical failure or a normal result. These features eventually translate into under-recognition of sAML patients and lead to inadequate clinical management, since this highrisk population deems intensive treatment strategies comprising the administration of innovative agents or allocation to clinical trials, which are potentially able to improve the rates of complete remission (CR), ${ }^{4-7}$ followed by a rapid consolidation with allogeneic hematopoietic stem cell transplant (alloHSCT), ${ }^{7,8}$ Therefore, more accurate diagnostic approaches are warranted. In this regard, studies focusing on the molecular landscape of sAML and preceding conditions have suggested the possibility of defining distinct subtypes of AML based on their mutational profiles. Mutations in genes involved in chromatin regulation (ASXL1, EZH2, BCOR, STAG2) and RNA splicing (SRSF2, SF3B1, U2AF1, ZRSR2) have shown high specificity for sAML after MDS; ${ }^{9}$ these mutations, called secondary-type mutations, occur early in leukemogenesis (likely representing the expansion of clones acquired during previous $\mathrm{MDS})^{10,11}$ and often persist in clinical remission, presenting as constitutively chemoresistant. Although less characterized, sAML cases progressing after MPN display similar features. ${ }^{12,13}$ However, a closely related mutational signature can be identified also in some de novo AML cases. ${ }^{14,15}$ In the seminal study conducted by Papaemmanuil et al. ${ }^{14}$ on a large cohort of AML patients, overlapping mutations in genes regulating RNA splicing (SRSF2, SF3B1, U2AF1, and ZRSR2), chromatin (ASXL1, STAG2, BCOR, KMT2A-PTD, EZH2, and PHF6), or transcription (RUNX1) constituted an independent genomic class, called the chromatin-spliceosome group. Mutations in RUNX1 and KMT2A-PTD frequently cooccurred with other mutations of the signature and, albeit not universally observed in sAML, have been reported as more significantly associated with high-risk than low-risk $\mathrm{MDS},{ }^{11}$ and consistently occurred at intermediate time points and not as founding mutations. ${ }^{11,14-16}$ Strikingly, $91 \%$ of patients in this group were clinically defined as de novo AML; although heterogeneous, mutations in this molecular signature were consistently associated with older age, lower white blood cell and blast counts, lower rates of response to induction chemotherapy and higher relapse rates. While these results warrant prospective validation, it can be hyphothesized that the presence of these mutations represent the trace of a previous, unrecognized MDS or MPN phase. However, a formal comparison between de novo AML patients carrying chromatinspliceosome (CS) mutations and sAML patients defined by standard criteria, along with associated outcomes, is currently lacking.

In order to investigate the clinical significance of the CS mutational signature, we reassessed patients' diagnosis according to the presence of CS mutations in a large cohort of newly diagnosed AML patients enrolled into a prospective trial (NILG AML 02/06) (clinicaltrials gov. Identifier: NCT00495287). ${ }^{17}$ In this study, we report the characteristics and outcomes of initially defined de novo AML patients carrying CS mutations as compared with other clinically defined de novo AML patients without CS mutations and patients with sAML defined by standard WHO criteria.

\section{Methods}

\section{Patients, treatment, cytogenetic and molecular analyses}

The NILG-AML 02/06 multi-center Italian trial ${ }^{17}$ enrolled 574 patients with newly diagnosed AML ( $\geq 20 \%$ bone marrow [BM] blasts) or high-risk MDS (10-19\% BM blasts) between 2007 and 2012. All participants were randomized to receive induction with standard-dose idarubicin, cytarabine and etoposide (ICE) or high-dose cytarabine and idarubicin (sHD). Patients not responding to first induction underwent an intensified re-induction with sHD. Consolidative alloHSCT was performed in highrisk patients based on study-specific risk stratification, as previously reported. ${ }^{17}$ Written informed consent for inclusion in the clinical trial and genetic analyses was provided by all patients. Study protocols were in accordance with the Declaration of Helsinki and approved by the Institutional Review Boards of each participating center.

Informative karyotype was locally obtained at diagnosis for 413 patients. Molecular analyses were centrally performed on samples collected at diagnosis (see the Online Supplementary Methods). NPM1, FLT3-ITD and point mutations, RUNX1RUNX1T1, CBFb-MYH11, biallelic CEBPa and KMT2A-PTD mutations were tested on all patients using polymerase chain reaction (PCR), Sanger sequencing and/or fragment analysis. Targeted next-generation sequencing (NGS) was performed on 196 normal karyotype patients using an amplicon-based method (Trusight Myeloid, Illumina, San Diego, California, USA) $(\mathrm{n}=161)$ amplifying 54 gene regions and a capture-based method (Sophia Myeloid Solution, Sophia Genetics SA, Saint Sulpice, Switzerland) $(\mathrm{n}=35)$ selecting 30 gene regions (Online Supplementary Tables $S 1$ and S2). ${ }^{18}$

In order to confirm our results in an independent cohort, we also evaluated a single-center series of AML patients $(n=50)$ treated at ASST Ospedale Papa Giovanni XXIII between 2012 and 2020 .

\section{Definition of acute myeloid leukemia categories}

The following AML categories were defined among patients enrolled into the trial: i) CS-AML: with the specific goal of validating the CS mutational signature, clinically defined de novo AML patients were included in this category based on the presence of at least one variant described according to Papaemmanuil et al. ${ }^{14}$ including ASXL1, STAG2, BCOR, EZH2, PHF6, SRSF2, SF3B1, U2AF1, ZRSR2, RUNX1 and KMT2A-PTD (or cytogenetic alterations in KMT2A gene in the $11 \mathrm{q} 23$ cytogenetic region), excluding patients with concurrent WHO-recur- 
rent abnormalities; ${ }^{1}$ ii) sAML: patients with a documented clinical history of MDS, MDS/MPN or MPN and/or cytogenetic WHO criteria of AML with MDS-related changes; ${ }^{1}$ iii) de novo AML: none of the above. In order to avoid any ambiguous interpretation, morphological WHO criteria of AML with MDS-related changes were not considered in the definition of sAML. Patients with MDS, therapy-related AML or not provided with a cytogenetic and/or molecular characterization were excluded from the analysis.

\section{Statistical analysis}

The clinical endpoints of the study were defined according to standard criteria. ${ }^{19}$ Comparisons between baseline characteristics and AML categories were analyzed using the MannWhitney $U$ test for countinuous and Chi-squared or Fisher's exact test for categorical variables. Overall survival (OS) and disease-free survival (DFS) were estimated by the Kaplan-Meier method and any differences between AML categories or consolidation treatment were evaluated with log-rank test. Cox models were used to estimate hazard ratios (HR) with 95\% Confidence Intervals (CI) in univariate and multivariable analysis on survival outcomes. AlloHSCT was considered as a time- dependent event; outcome data were estimated by the MantelByar method and graphically illustrated by Simon-Makuch plots. All reported $P$-values are two-sided and set at $5 \%$ significance level. All analyses were performed with $\mathrm{R}$ software, version 3.5.0.

\section{Results}

\section{Characteristics of patients}

Among 574 adult patients enrolled in the NILG-AML 02/06 trial, ${ }^{16} 413(72 \%)$ with full genetic characterization resulted evaluable and were classified as $55 \mathrm{CS}-\mathrm{AML}$ patients, 100 sAML patients (28 defined by clinical history, of which 24 after MDS or MDS/MPN and four after MPN, and 72 defined by cytogenetic criteria) and 258 de novo AML patients (Figure 1). The 55 cases reclassified as CS-AML represented $17.6 \%$ of otherwise defined de novo AML patients and $13 \%$ of the whole analyzed cohort.

The main clinical characteristics of patients are reported in Table 1. Compared to de novo AML, patients with sAML and CS-AML were similarly older (median age 48,

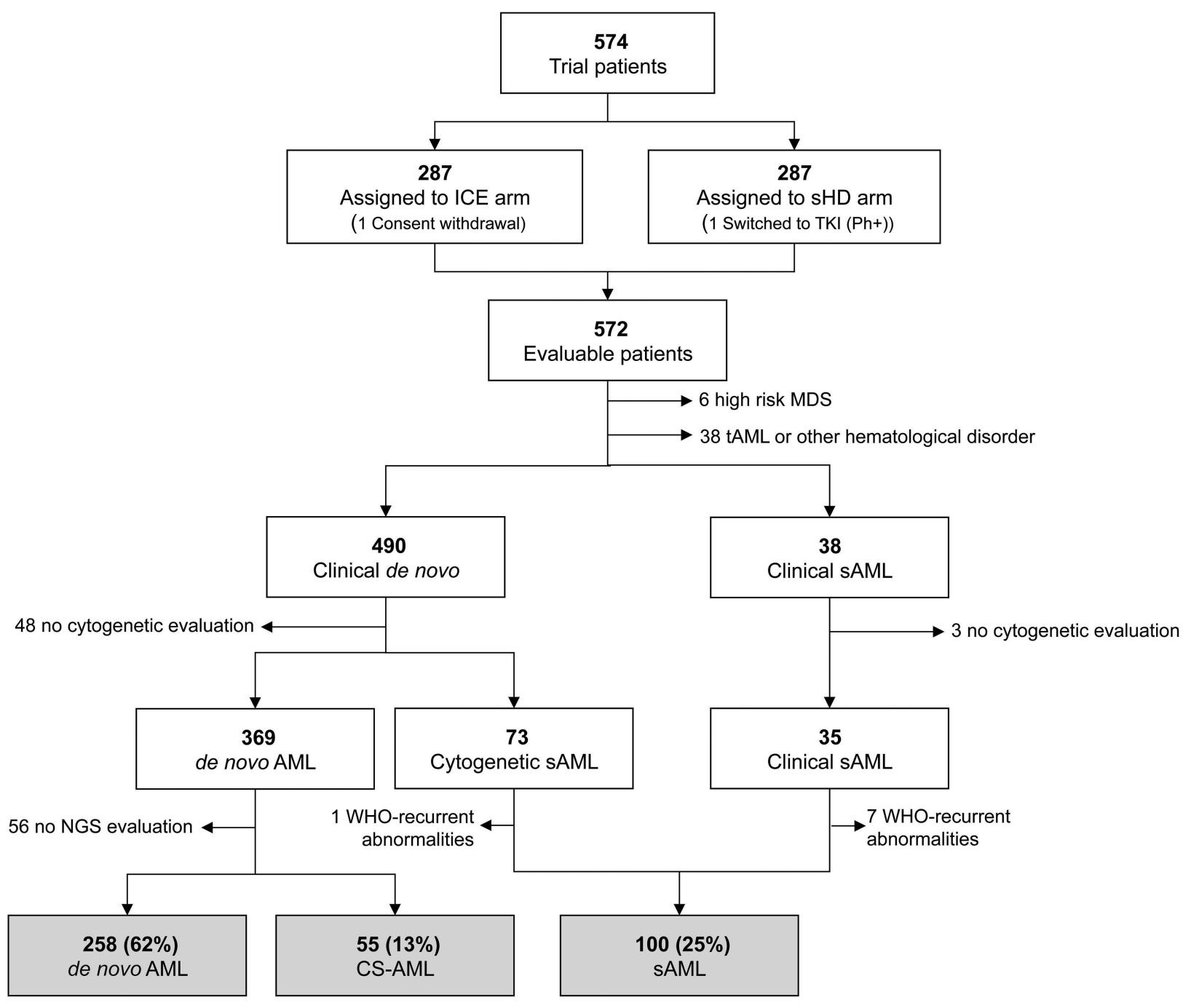

Figure 1. CONSORT diagram illustrating patient selection. ICE: idarubicin, cytarabine, etoposide; sHD: sequential high-dose chemotherapy; TKI: tyrosine-kinase inhibitors; AML: acute myeloid leukemia; tAML: therapy-related AML; sAML: secondary AML; CS-AML: de novo AML carrying chromatin-spliceosome mutations; MDS: myelodysplastic syndrome; WHO: World Health Orginization; NGS: next-generation squencing. 
59 and 58 years respectively, $P<0.0001)$ and presented at diagnosis with lower white blood cell counts (WBC) $(P<0.0001)$, with no significant differences between the two latter categories. A lower BM blast infiltration was reported in SAML as compared with both de novo AML $(P<0.0001)$ and CS-AML $(P=0.02)$. By morphological analysis, multilineage dysplasia was described at diagnosis in a minor proportion of CS-AML patients $(11 \%)$, close to that of sAML patients $(9 \%, P=0.77)$ and similarly higher than that of de novo AML patients $(2 \%, P=0.0051)$.

The cytogenetic and molecular characteristics of the cohort are summarized in Figure 2 and the Online Supplementary Table S2.

In the CS-AML category $(n=55)$ (Figure 2A and B), the majority of patients $(87 \%)$ had a normal karyotype; 160 mutations were found in total, with a median of three mutations per patient (range, 1-6). The most frequently reported mutations of the CS signature were in KMT2A (KMT2A-PTD), RUNX1 and ASXL1 genes (respectively $45.5 \%, 44.4 \%$ and $22.2 \%$ of evaluable patients), while other mutations accounted for $5-17.5 \%$ of cases. While $54.5 \%$ of patients in this category presented with a single CS mutation, overlap and significant associations between CS mutations were observed in $45.5 \%$ of cases. Other mutations scored in CS-AML patients included IDH2, DNMT3A, FLT3-ITD, TET2 and NRAS (respectively $20 \%, 15.6 \%, 14.5 \%$ and $11 \%$ of evaluable patients), and others (Figures 3 and 4). We also included two patients with previously undescribed variants (UPN 633, STAG2 p.Met135Ile and UPN 753, SRSF2 p.Val18Leu) in the CS-AML category due to their predicted pathogenicity. One more patient harboring the RUNX1 p.Ala60V variant (UPN 3) was also included. This variant has been found in familial platelet disorder associated to myeloid malignancy and is reported as such in the ClinVar database (https://www.ncbi.nlm.nih.gov/clinvar/variation/ $\mathrm{V}$ CV000463986).

Among the 100 sAML patients (Figure 2C and D), a huge proportion carried a complex karyotype (58\%) and abnormalities of chromosome $7(12 \%)$ or $5(5 \%)$. Nevertheless, $13 \%$ of patients in this category had a normal karyotype; 62 mutations were reported in total (median 0, range 0-8), mostly involving CS genes.

The de novo AML category $(\mathrm{n}=258)$ (Figure $2 \mathrm{E}$ and $\mathrm{F}$ ) included $25 \%$ of patients with a core binding factor AML, a high prevalence $(65 \%)$ of patients with normal karyotype and $10 \%$ of patients with other non WHO-recurrent cytogenetic abnormalities. A total of 607 mutations were scored (median two per patient, range 0-15), the most frequently represented being NPM1 $(60.7 \%$ of patients), DNMT3A (49.3\%) and FLT3-ITD (27.5\%).

\section{Outcomes after intensive induction}

Patients with de novo AML, sAML and CS-AML were equally distributed among the randomly assigned induction regimen (conventional ICE or high-dose sHD) and showed a comparable performance status (Table 1).

After the first induction cycle, a lower proportion of sAML patients achieved CR (51 of 100, 51\%) as compared to both de novo AML (228 of $258,88.4 \% ; P<0.0001)$ and CS-AML (43 of 55, 78.2\%; $P=0.002$ ). However, also CS-AML showed a trend toward an inferior CR rate as compared to de novo AML $(P=0.07)$, although this did not reach statistical significance, eventually due to the relative small number of patients in this group. Interestingly, no significantly worse CR rate was observed between

Table 1. Demographic and clinical characteristics of patients by acute myeloid leukemia category.

\begin{tabular}{|c|c|c|c|c|c|c|}
\hline Clinical characteristics & $\begin{array}{l}\text { CS-AML } \\
N=55\end{array}$ & P* & $\begin{array}{c}\text { SAML } \\
N=100\end{array}$ & $P^{* *}$ & $\begin{array}{c}\text { de novo AML } \\
N=258\end{array}$ & $P^{* * *}$ \\
\hline $\begin{array}{l}\text { Age [years], median (range) } \\
\quad<60, \mathrm{n}(\%) \\
\quad \geq 60, \mathrm{n}(\%)\end{array}$ & $\begin{array}{l}58(20-72) \\
33(60) \\
22(40)\end{array}$ & $\begin{array}{l}0.5542 \\
0.4015\end{array}$ & $\begin{array}{l}59(22-72) \\
53(53) \\
47(47)\end{array}$ & $\begin{array}{l}<0.0001 \\
<0.0001\end{array}$ & $\begin{array}{l}48(16-73) \\
207(80.2) \\
51(19.8)\end{array}$ & $\begin{array}{l}0.0001 \\
0.0013\end{array}$ \\
\hline $\begin{array}{l}\text { Sex, n (\%) } \\
\text { M } \\
\text { F }\end{array}$ & $\begin{array}{l}25(45.5) \\
30(54.5)\end{array}$ & 0.0349 & $\begin{array}{l}63(63) \\
37(37)\end{array}$ & 0.0076 & $\begin{array}{l}122(47.3) \\
136(52.7)\end{array}$ & 0.8048 \\
\hline $\begin{array}{l}\text { ECOG PS, n (\%) } \\
\quad 0-1 \\
2-3\end{array}$ & $\begin{array}{c}49(89.1) \\
6(10.9)\end{array}$ & 0.5674 & $\begin{array}{l}92(92) \\
8(8)\end{array}$ & 0.4207 & $\begin{array}{l}230(89.1) \\
28(10.9)\end{array}$ & 0.9903 \\
\hline Hepatomegaly, n (\%) & $5(9.1)$ & 0.5216 & $6(6)$ & 0.4916 & $21(8.1)$ & 0.7897 \\
\hline Splenomegaly, n (\%) & $2(3.6)$ & 0.2150 & $10(10)$ & 0.3159 & $36(14)$ & 0.0334 \\
\hline Extramedullary involvement, n (\%) & $6(10.9)$ & 0.5458 & $7(7)$ & 0.0267 & $41(15.9)$ & 0.3477 \\
\hline Hemoglobin $[\mathrm{g} / \mathrm{dL}]$, median (range) & $9.3(5.1-13.8)$ & 0.6334 & $8.8(4.3-13.7)$ & 0.1905 & $9.3(3-15.8)$ & 0.7040 \\
\hline WBC count $\left[\times 10^{9} / L\right]$, median (range) & $8.1(1.1-252)$ & 0.1794 & $4.8(0.8-237)$ & $<0.0001$ & $22.3(0.5-282)$ & 0.0004 \\
\hline Platelets, median (range) & $77(12-815)$ & 0.1441 & $57(2-338)$ & 0.3649 & $52(5-852)$ & 0.0151 \\
\hline BM blast cells (\%), median (range) & $80(8-100)$ & 0.0227 & $64(2-100)$ & $<0.0001$ & $80(0-100)$ & 0.2067 \\
\hline AML with multilineage dysplasia, $\mathrm{n}(\%)$ & $6(10.9)$ & 0.7786 & $9(9)$ & 0.0041 & $5(1.9)$ & 0.0051 \\
\hline Induction treatment, $\mathrm{n}(\%)$ & & 0.7613 & & 0.6852 & & 0.5065 \\
\hline ICE & $25(45.5)$ & & $48(48)$ & & $130(50.4)$ & \\
\hline sHD & $30(54.5)$ & & $52(52)$ & & $128(49.6)$ & \\
\hline
\end{tabular}

CS-AML: chromatin-spliceosome acute myeloid leukemia; sAML: secondary acute myeloid leukemia; de novo AML: de novo acute myeloid leukemia; ECOG PS: Eastern Cooperative Oncology Group Performance Status; WBC: white blood cell count; BM: bone marrow; ICE: idarubicin, cytarabine and etoposide; sHD: sequential high-dose chemotherapy cytarabine and idarubicin. $P$-values refer to: *CS-AML $v$ s. sAML; **AML $v$ s. de novo AML; ** * de novo AML $v$ s. CS-AML. Hepatomegaly was defined as lower liver edge $>2 \mathrm{~cm}$ from costal margin. Splenomegaly was defined as spleen $>1 \mathrm{~cm}$ from costal margin, confirmed by ultrasound scan with longitudinal axis $>12 \mathrm{~cm}$. Extramedullary AML was defined as AML presenting with central nervous system involvement or mass lesions. 
these latter groups $(P=0.14)$ when accounting for both first induction (performed in all patients) and intensified re-induction (performed in patients who did not respond after first cycle). By contrast, sAML patients did not improve their CR rate $(59 \%)$ even after undergoing reinduction, as compared with both de novo $\mathrm{AML}$ (92.6\%,
$P<0.0001)$ and CS-AML (85.5\%, $P=0.001)$. In addition, early death more frequently occurred in sAML patients (14 of $100,14 \%$ ) than in de novo AML (11 of $258,4.3 \%$; $P=0.003$ ) and CS-AML (three of $55,5.5 \% ; P=0.17$ ). Globally, $41 \%$ of sAML patients, $14.5 \%$ of CS-AML patients and $7.3 \%$ of de novo AML patients did not
A

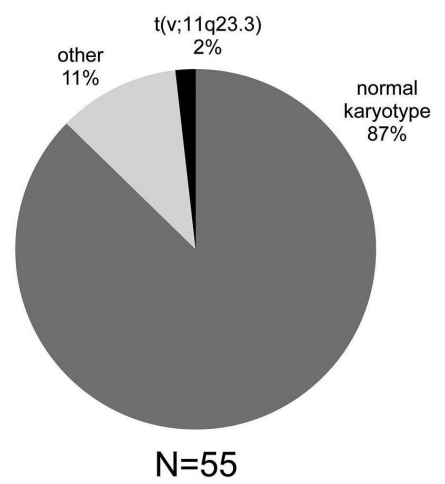

C

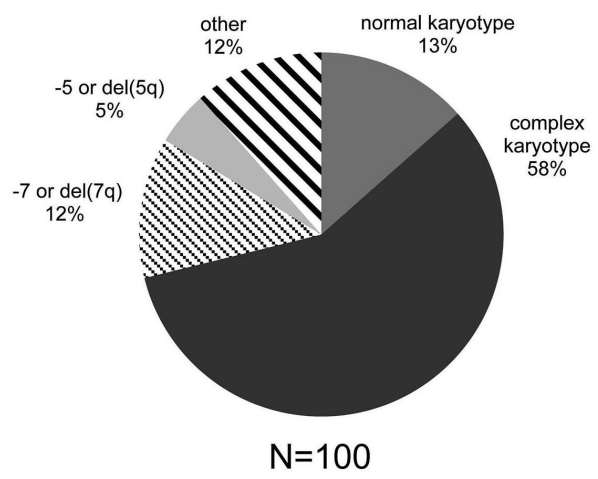

E de novo AML

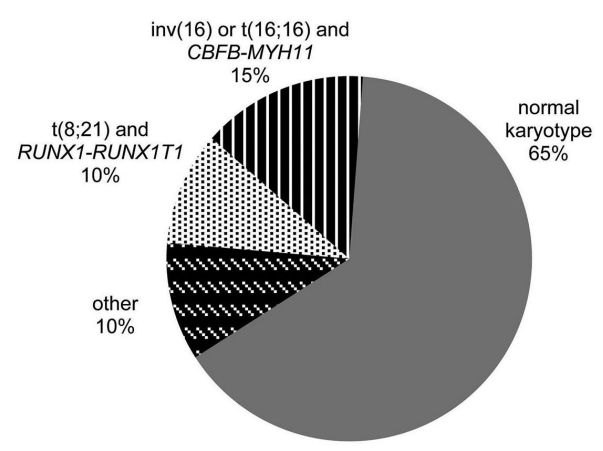

B

D

$\mathbf{F}$
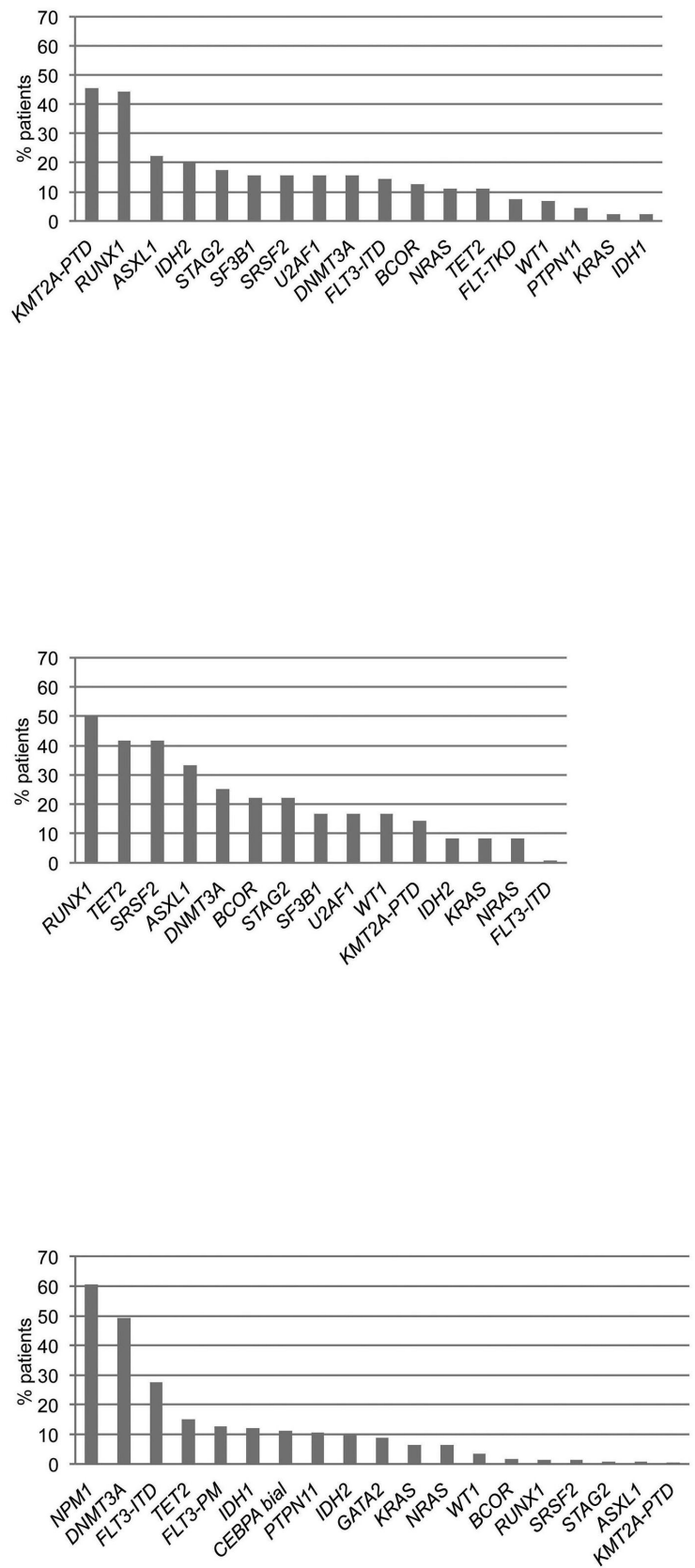

Figure 2. Cytogenetic and molecular characteristics of acute myeloid leukemia categories. For each acute myeloid leukemia (AML) category, pie charts depict the distribution of chromosomal abnormalities, while histograms show the frequency of individual mutations. (A and B) CS-AML (de novo AML carrying chromatin-spliceosome mutations), ( $C$ and $D)$ secondary AML (SAML) and (E and F) de novo AML. The label "other" includes: for the CS-AML category, abnormalities of chromosome 11 [(other than $t(v ; 11 q 23.3)$ and del(11q)] and +8; for the sAML category, del(11q), +8, del(12p), t(5q;12p), t(1p;3q), t(3q;5q) and -Y; for the de novo AML category, +8, del(9q), +21, monosomy 21, +13, t(8q;11q), inv(3), monosomy $X,-Y$, del(16q), add(4q), add(6p), t(13p;17p). 
achieve CR because of early death or chemoresistance; these patients had a dismal 1-year OS (17\%) (Online Supplementary Figure S1).

We investigated factors affecting the probability of achieving CR (accounting for both induction cycles) by performing univariate analysis within each AML category. In de novo AML, a significantly negative impact was observed for advanced age, impaired performance status, high WBC and the presence of FLT3-ITD mutations. Apart from the presence of SRSF2 mutations among CS-

Table 2. Multivariable analysis for complete remission, overall survival and disease free survival on the whole patient cohort.

\begin{tabular}{|c|c|c|c|c|c|c|}
\hline All patients & Complete & & Overa & & Disease-ir & ival \\
\hline Characteristics & HR (95\% CI) & $P$ & HR (95\% CI) & $P$ & HR (95\% Cl) & $P$ \\
\hline $\begin{array}{l}\text { Age [years] } \\
\geq 60\end{array}$ & $0.38(0.21-0.7)$ & 0.0017 & $1.67(1.25-2.23)$ & 0.0005 & $1.32(0.95-1.83)$ & 0.1030 \\
\hline $\begin{array}{l}\text { ECOG PS } \\
2-3\end{array}$ & $0.25(0.11-0.57)$ & 0.0010 & $2.32(1.55-3.47)$ & $<0.0001$ & $1.46(0.87-2.43)$ & 0.1502 \\
\hline $\begin{array}{l}\text { WBC count }\left[\times 10^{\circ} L\right] \\
\quad \geq 50\end{array}$ & $0.5(0.24-1.03)$ & 0.0582 & $1.69(1.23-2.3)$ & 0.0010 & $1.59(1.14-2.21)$ & 0.0063 \\
\hline $\begin{array}{l}\text { Induction arm } \\
\text { ICE }\end{array}$ & $0.87(0.48-1.57)$ & 0.6486 & $1.27(0.97-1.66)$ & 0.0856 & $1.27(0.95-1.7)$ & 0.1046 \\
\hline $\begin{array}{l}\text { AML category } \\
\text { SAML } \\
\text { CS-AML }\end{array}$ & $\begin{array}{c}0.09(0.05-0.19) \\
0.51(0.2-1.37)\end{array}$ & $\begin{array}{c}<0.0001 \\
0.1608 \\
\end{array}$ & $\begin{array}{c}3.71(2.69-5.12) \\
2.2(1.48-3.25) \\
\end{array}$ & $\begin{array}{l}<0.0001 \\
0.0001\end{array}$ & $\begin{array}{l}2.54(1.76-3.67) \\
1.89(1.27-2.81)\end{array}$ & $\begin{array}{r}<0.0001 \\
0.0018\end{array}$ \\
\hline
\end{tabular}

WBC: white blood cell;ECOG PS: Eastern Cooperative Oncology Group Performance Status; SAML: secondary acute myeloid leukemia; ICE: idarubicin, cytarabine and etoposide; HR: hazard ratio; CI: Confidence Interval; CS: chromatin-spliceosome; CS-AML: de novo AML carrying the CS mutations.

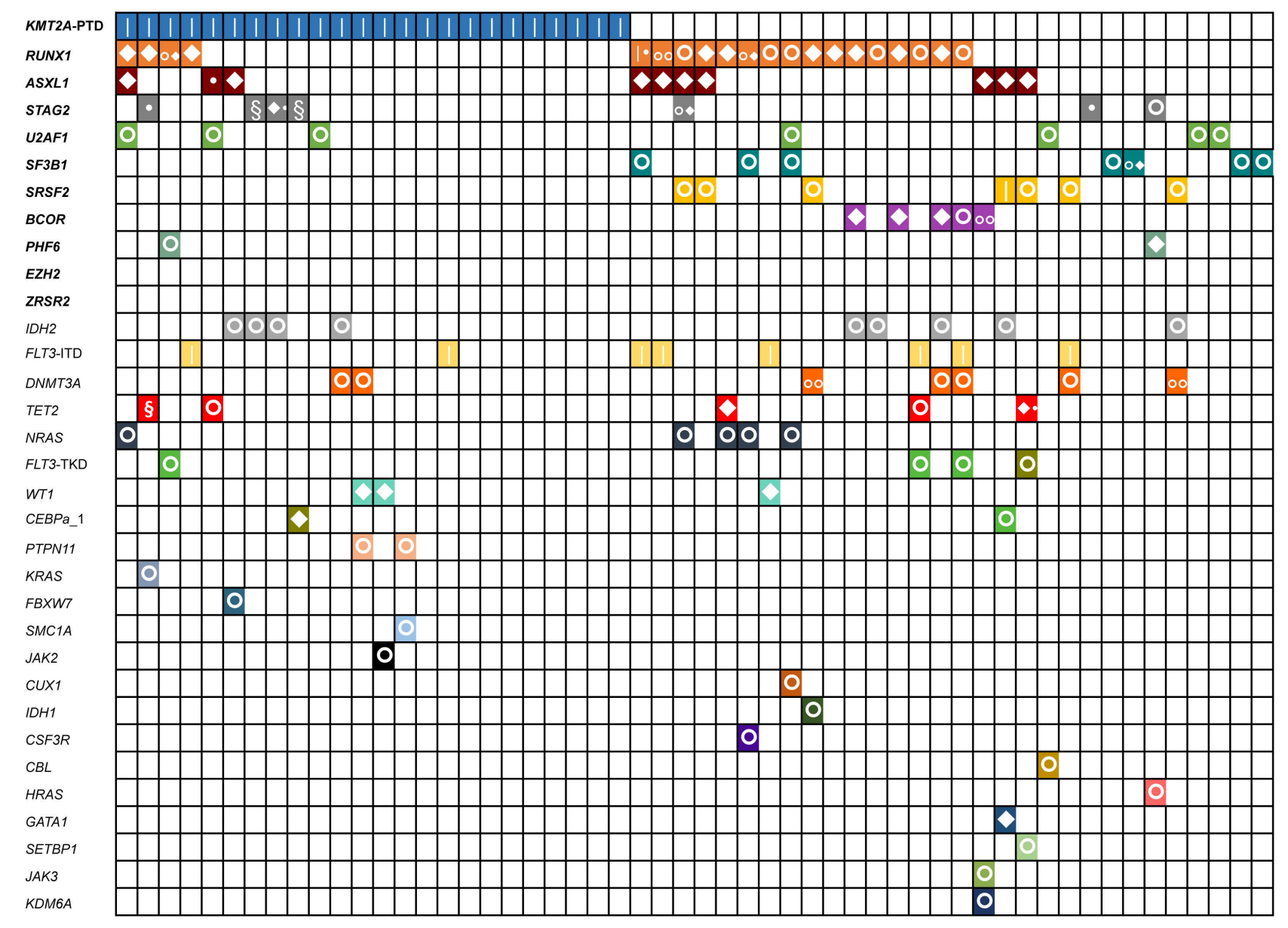

Figure 3. Mutational profile of acute myeloid leukemia patients carrying the chromatin-spliceosome mutation. Each column represents an individual acute myeloid leukemia patient carrying a chromatin-spliceosome mutation (CS-AML patient), while each row represents a single gene mutation out of the list at the left. Colored bars indicate the presence of one or more mutations of each gene. Variant types are specified according to the legend at the bottom of the figure.

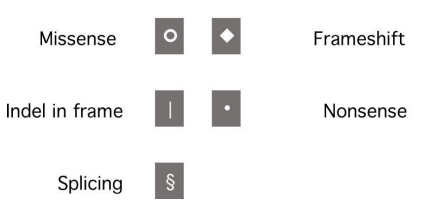


AML patients (HR 0.16; 95\% CI: 0.02-1.02; $P=0.05$ ), no other relevant clinical or biological factors (including the intensity of induction regimen) were identified for sAML and CS-AML patients (Online Supplementary Table S3).

\section{Survival outcomes}

The median follow-up for survival analysis was 4.9 years (range, 0.2-8.4). De novo AML patients showed a markedly better 5 -years OS (61\%) and DFS (54\%) than CS-AML (OS 30\%, DFS 26\%; $P<0.0001$ and $P=0.0009$ respectively) and sAML (OS $17 \%$, DFS $22 \% ; P<0.0001$ for both comparisons) (Figure $5 \mathrm{~A}$ and $\mathrm{B}$ ). Patients with sAML had a significantly worse OS as compared to CSAML $(P=0.02)$ (Figure 5A); however, possibly due to the high mortality rate of non-responding patients, no difference was observed between the two categories when considering only patients achieving CR, who showed an overlapping 5 -years DFS $(26 \%$ and $22 \%$ respectively, $P=0.32$ ) (Figure $5 \mathrm{~B}$ ). A similar trend of $\mathrm{OS}$ according to the respective categories was confirmed on a smaller $(\mathrm{n}=50)$, independent single-center cohort (Online Supplementary Figure S2; for demographic and clinical characteristics of this cohort, see the Online Supplementary Table S6).

According to trial risk stratification criteria, consolidative alloHSCT in first CR was administered in 18 of 47 (38.3\%) CS-AML, 31 of 59 (52.5\%) sAML and 80 of 239 $(33.5 \%)$ de novo AML patients, at a median age of $49.5,52$ and 44.5 years, respectively. By time-dependent analysis, the administration of alloHSCT in first CR carried a 5years survival advantage in each AML category (CS-AML: $48 \%$ vs. $24 \%, P=0.07$; sAML: $38 \%$ vs. $8 \%, P=0.0001$; de novo AML: $75 \%$ vs. $59 \%, P=0.01$ ) (Figure $6 \mathrm{~A}$ to $\mathrm{C}$ ).

By multivariable analysis performed on the whole patients' cohort accounting for age, performance status, WBC count at diagnosis and induction arm (standard vs. high-dose regimen), the markedly unfavorable prognosis of the sAML category was evident for each considered outcome (CR: HR 0.09, 95\% CI: 0.05-0.19, P<0.0001; OS: HR 3.71, 95\% CI: 2.69-5.12, $P<0.0001$; and DFS: HR 2.54, $95 \%$ CI: $1.76-3.67, P<0.0001)$. Although to a lesser degree, also the CS-AML category was independently associated to a negative prognosis, in terms of OS (HR 2.2 , 95\% CI: 1.48-3.25, $P=0.0001)$ and DFS (HR 1.89, 95\% CI: $1.27-2.81, P=0.0018)$, but not CR (Table 2). Other factors affecting clinical outcomes included age $\geq 60$ years, performance status (on CR and OS) and WBC $\geq 50 \times 10^{\circ} / \mathrm{L}$ (on OS and DFS).

Since CS mutations frequently co-occurred within individual patients, we sought to investigate whether specific variants of the signature might be independently responsible for the adverse prognosis of CS-AML patients. In a multivariable analysis performed on the CS-AML cohort including CS-mutations and adjusting for the same variables as in previous analysis (Online Supplementary Table S4), only RUNX1 and U2AF1 independently affected OS (HR 3.55, 95\% CI: 1.28-9.87, $P=0.01$; and HR 6.87, 95\% CI: $1.71-27.55, P=0.006$ ) and DFS (HR 3.13, 95\% CI: $1.1-8.95$, $P=0.03$; and HR 16.46, 95\% CI: 3.14-86.31, $P=0.0009$ ). Notwithstanding, even after subtracting $R U N X 1$ and/or $U 2 A F 1$-mutated patients from the first multivariable analysis (Online Supplementary Table S5), the CS-AML category maintained indipendently worse OS (HR 1.83, 95\% CI: 1.07-3.14, $P=0.0281$ ) and DFS (HR 1.94, 95\% CI: 1.15-3.26, $P=0.0126)$ as compared to de novo AML.

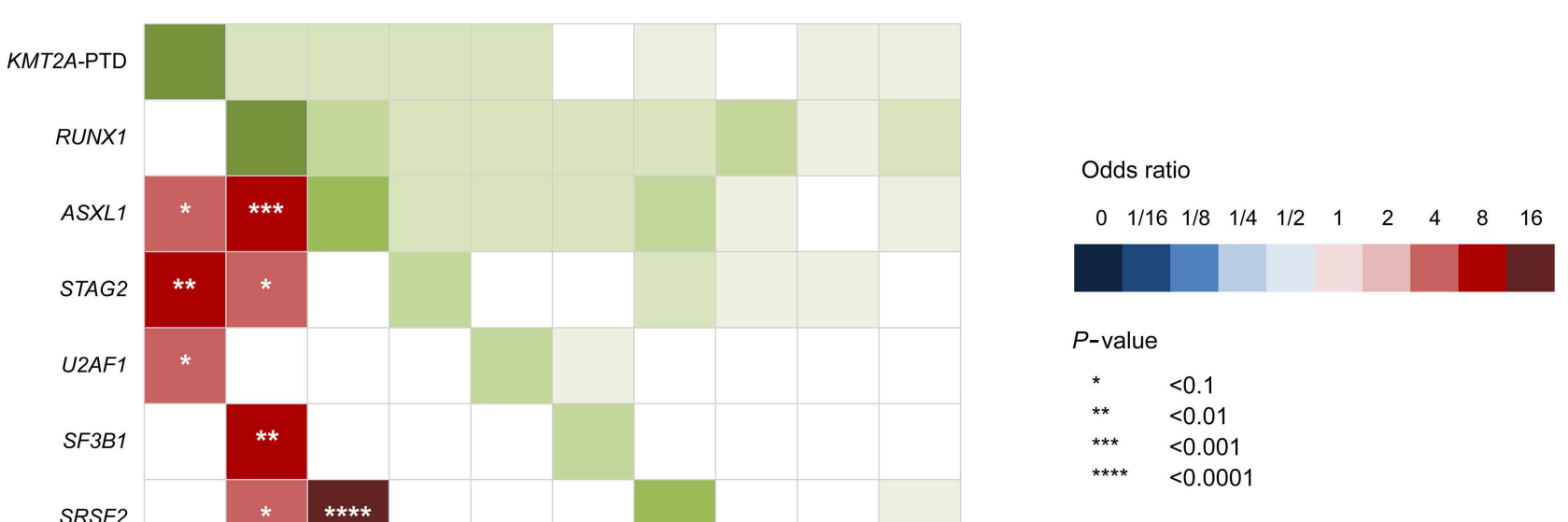

Occurrence of pairwise associations

$\begin{array}{lllllll}1 & 5 & 10 & 20 & 50 & 100 & 200\end{array}$

ZRSR2
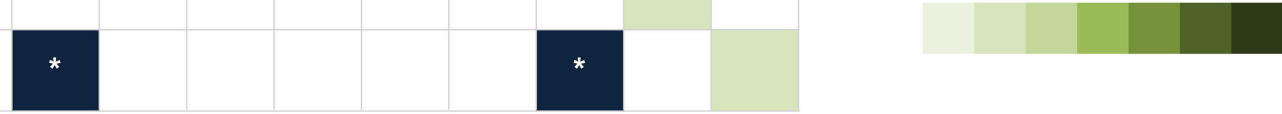

KMT2A-PTD RUNX1 ASXL1 STAG2 U2AF1 SF3B1 SRSF2 BCOR PHF6 ZRSR2

Figure 4. Patterns of co-occurrence and mutual exclusivity of gene mutations among 55 CS-AML patients. In the lower triangle are shown pairwise associations between gene mutations. For each pair, odds ratios indicate an in-creased $(>1)$ or decreased $(<1)$ probability of co-occurrence between the two mutations as assessed by the Fisher exact test for statistical significance. The odds ratio of the association is color coded and the significance level is indicated by the number of asterisks in each colored square as reported in the legend at the right of the figure. The upper triangle illustrates the absolute number of occurrences of each molecular pair, shown in green gradient and divided in intervals as reported in the legend. The analysis was performed on the whole study cohort ( $\mathrm{n}=413$ ), excluding mutations occurring in less than six patients and not defining AML categories. 


\section{Discussion}

In this study, we challenged a CS-mutational signature ${ }^{14}$ against conventionally defined sAML and de novo AML patients to seek whether a comparison between related outcomes might provide a basis for implementing current clinical and cytogenetic diagnostic criteria of sAML with molecular information. Importantly, the identification of

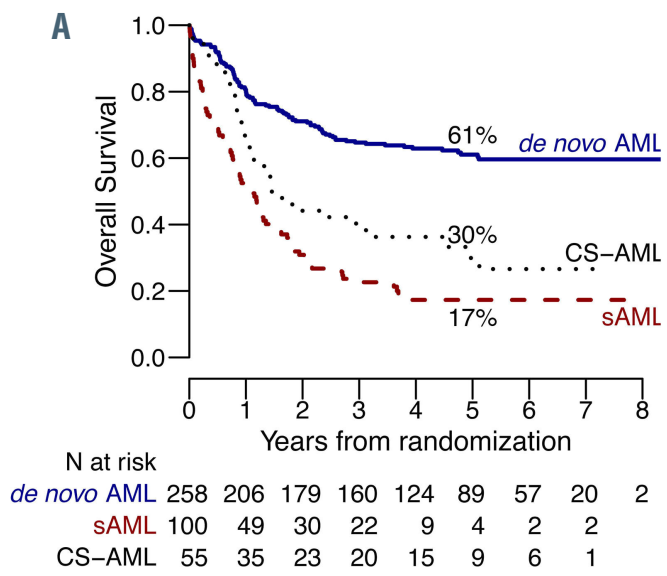

patients carrying CS mutations has been allowed by conventional methods and by commercially available NGS solutions, which can be easily and cost-effectively implemented in the routine diagnostic work-up of AML. ${ }^{20}$ Since diagnostic uncertainty is higher in cases lacking informative cytogenetics, we focused the search of CS mutations on patients with normal karyotype as these mutations were infrequently associated with abnormalities of chro-

B

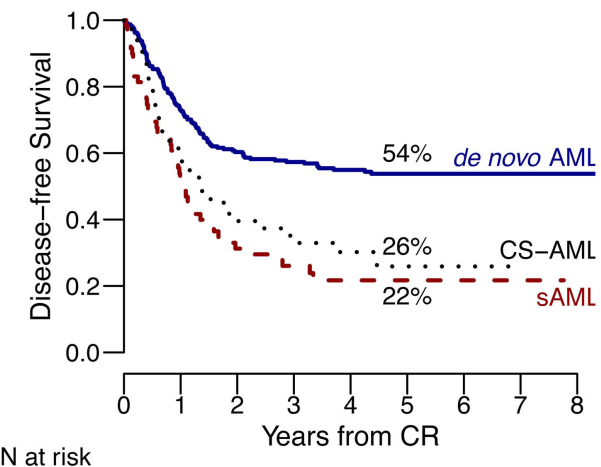

$\begin{array}{lllllllll}\text { novo AML } 239 & 173 & 142 & 132 & 100 & 74 & 47 & 18 & 2\end{array}$

$\begin{array}{lllllllll}\text { SAML } & 59 & 30 & 18 & 15 & 6 & 3 & 2 & 2\end{array}$

CS-AML $\begin{array}{llllllll}47 & 27 & 18 & 15 & 9 & 6 & 5 & 1\end{array}$

Figure 5. Kaplan-Meier survival analysis according to acute meloid leukemia category. Survival estimates were calculated at 5 years and not censored at allogeneic transplant. (A) Overall survival; CS-AML vs. de novo AML, $P<0.0001$; SAML vs. de novo AML, $P<0.0001$; CS-AML vs. SAML, $P=0.02$. (B) Disease free survival; CSAML vs. de novo AML, $P=0.0009$; sAML vs. de novo AML, $P<0.0001$; CS-AML vs. sAML, $P=0.32$. CS: chromatin-spliceosome; AML: acute myeloid leukemia; sAML: secondary AML; CS-AML: de novo AML carrying the CS mutations.
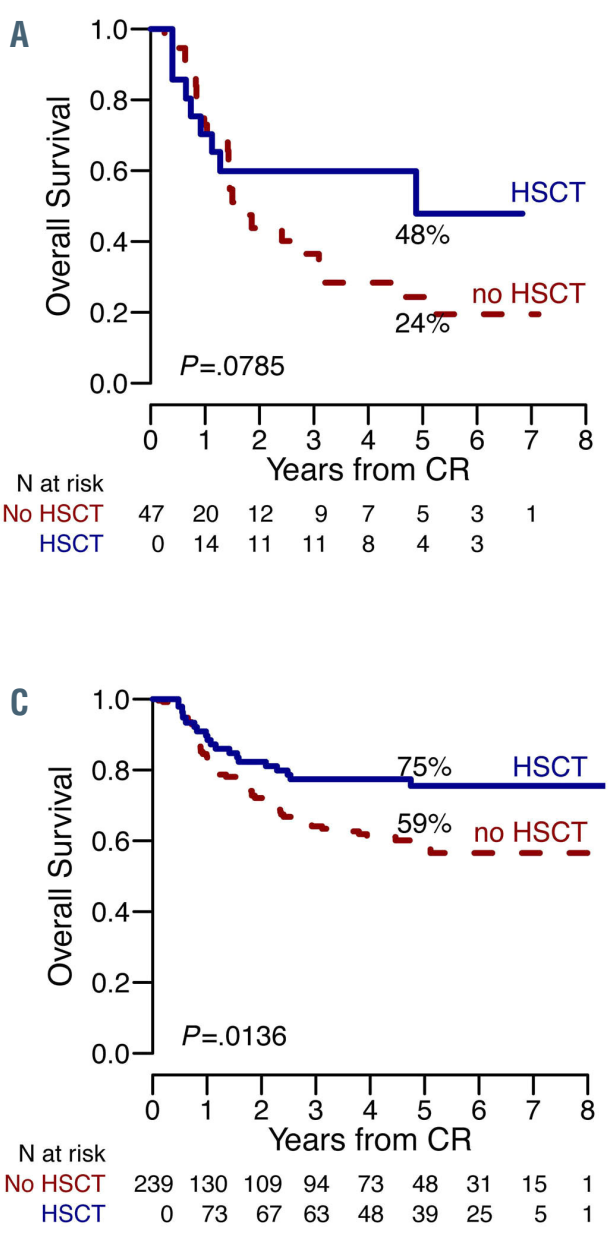

B

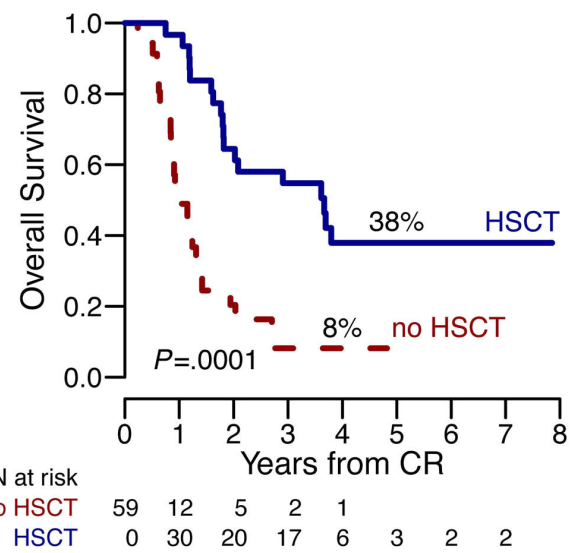

Figure 6. Simon-Makuch plots of overall survival according to allogeneic hematopoietic stem cell transplant. Transplant was considered as a timedependent event. Survival estimates were calculated at 5 years from the date of complete remission (CR) after induction chemotherapy. (A) CS-AML, (B) SAML and (C) de novo AML. CS: chromatin-spliceosome; AML: acute myeloid leukemia; sAML: secondary AML; CS-AML: de novo AML carrying the CS mutations. 
mosomes 5 and 7 or complex karyotypes in previous studies. ${ }^{91}$ To the best of our knowledge, this is the first report formally comparing outcomes of patients with de novo AML carrying this mutational profile and sAML (defined by clinical and/or cytogenetic criteria) on a large multi-center prospective cohort. Patients included in the study showed a broad age range representative of the real life population, were homogeneously treated with intensive chemotherapy within a prospective clinical trial and had a long duration of follow-up, which allowed to effectively study the prognostic relevance of CS mutations. As an additional feature, we evaluated the impact of alloHSCT in each AML category.

In keeping with previous observations, the identification of a CS-mutational signature revealed markedly high-risk features in about $18 \%$ of otherwise defined de novo AML patients in our study, ${ }^{9,14,15}$ which represents a significant proportion of the whole analyzed cohort, quite consistent with other studies. ${ }^{14,22}$ Apart from mutations in KMT2A-PTD, RUNX1 and ASXL1, we showed that also mutations in U2AF1 carry an independent prognostic impact; of note, the adverse significance of CS mutations was maintained independently from RUNX1 and U2AF1, suggesting that the full signature might be further evaluated for the assignment to the high-risk group of the European LeukemiaNet (ELN) stratification model.' Based on these data, it remains unclear whether RUNX1-mutated AML, albeit constituting the most represented subgroup within this category, accounts for a separate clinical and prognostic entity.

In addition, we have highlighted that CS-AML more closely resembles sAML than de novo AML, in terms of clinical characteristics and outcomes. When considering OS, however, CS-AML stands as an intermediate category showing slightly but significantly better survival than sAML; this appears to be the consequence of the high rates of early chemoresistance and related mortality in sAML patients. A possible explanation for this difference resides in the negative impact of complex cytogenetics in the sAML group. Alternatively, this category might contain a mixture of both sAML and true de novo cases. As such, the role of the CS signature to accurately diagnose sAML remains not fully elucidated. However, from a practical point of view, the definition of CS-AML as a prognostically homogeneous group might be more clinically significant than a merely ontogenetic classification, as recently shown also for secondary-type mutations. ${ }^{23}$ In fact, since we observed a survival advantage in both SAML and CS-AML patients to whom alloHSCT was offered in first CR, patients with a CS molecular profile might be considered for intensive treatment strategies comprising rapid allocation to alloHSCT. The main challenge in this setting, however, would be the improvement of remission rates and depth by means of innovative therapeutics, possibly overcoming the inherent long-term chemoresistance of CS-AML and extending the access to a potentially curative alloHSCT. In this regard, considering the closer similitude between sAML and CS-AML might facilitate the optimization of available treatment strategies as well as the design of dedicated clinical trials. Among potentially useful agents, CPX-351 has been recently approved by the Food and Drug Administration and European Medicines Agency specifically for the treatment of AML with MDS-related changes or therapyrelated AML and might provide a similar benefit in fit CSAML patients. ${ }^{4}$ Furthermore, in a large phase Ib trial the anti-BCL-2 agent venetoclax in association with hypomethylating agents has provided promising CR and survival rates even in sAML patients or AML with poor cytogenetics, ${ }^{5}$ while spliceosome modulators ${ }^{24,25}$ and DOT1L inhibitors ${ }^{26}$ may represent a rational candidate for functional targeting of CS-AML and are currently evaluated in clinical trials involving myeloid neoplasms. Finally, in our dataset IDH2 mutations were reported in $20 \%$ of CS-AML patients, confirming previous observations ${ }^{14}$ and representing another potentially important therapeutic target in this population. ${ }^{27}$

The bottom line is that an accurate cytogenetic and molecular characterization is required at the diagnosis of AML, making it reasonable to wait for these data in order to perform the best treatment decision or enrollment into clinical trials; this approach has recently demonstrated to be safe in clinically stable patients. ${ }^{28}$ In this context, although conventional cytogenetics are still needed for a correct risk stratification, ${ }^{7}$ NGS technologies may overcome its limitations and long turnaround time, ${ }^{20}$ also providing additional information with improved cost effectiveness.

In conclusion, we have assessed the impact of a CSmutational signature on a large prospective cohort of AML patients employing a standardized, easily implementable NGS method and highlighting the need to detect this signature at diagnosis for an accurate risk prediction, with potentially relevant implications for the clinical management of AML patients.

\section{Disclosures}

No conflicst of interest to disclose.

\section{Contributions}

CC, FL and AR designed the research, analyzed and interpreted data, supervised the study and wrote the manuscript; $F D$, EO, TI, AG, GG, MF, DF, EA, ET, LDP, GR, EB, IC, MT, $A M S, D M, P C, L C, F C, M B, E T, A G, B F, R B$ performed research and collected data; genetic studies were performed by $R C, K B, L E, P Z, A M$; SS and OS performed and supervised genetic studies, collaborated on data interpretation, revised the manuscript and approved the final version; CP analyzed and interpreted data, performed statistical analysis and wrote the manuscript. All authors revised the manuscript and approved the final version before submission.

\section{Funding}

This work was partially supported by grants from Agenzia Italiana del Farmaco (Rome, Italy, Project FARM6YMY2N/2006), Fondazione Guido Berlucchi-Onlus (Brescia, Italy, 2006), and Associazione Italiana per la Ricerca sul Cancro (grant IG $2016 n$. 18568) to BF.

\section{References}

1. Arber DA, Orazi A, Hasserjian R, et al. The 2016 revision to the World Health Organization classification of myeloid neo- plasms and acute leukemia. Blood. 2016;127(20):2391-2405.

2. Weinberg OK, Seetharam M, Ren L, et al. Clinical characterization of acute myeloid leukemia with myel-odysplasia-related changes as defined by the 2008 WHO classification system. Blood. 2009;113(9):19061908

3. Naqvi K, Jabbour E, Bueso-Ramos C, et al. Implications of discrepancy in morphologic 
Clinical significance of chromatin-spliceosome AML

diagnosis of myelodysplastic syndrome between referral and tertiary care centers. Blood. 2011;118(17):4690-4693.

4. Lancet JE, Uy GL, Cortes JE, et al. CPX-351 (cytarabine and daunorubicin) liposome for injection versus conventional cytarabine plus daunorubicin in older patients with newly diagnosed secondary acute myeloid leukemia. J Chin Oncol. 2018;36(26):26842692.

5. DiNardo CD, Pratz K, Pullarkat V, et al. Venetoclax combined with decitabine or azacitidine in treatment-naive, elderly patients with acute myeloid leukemia. Blood. 2019;133(1):7-17.

6. Döhner H, Dolnik A, Tang L, et al. Cytogenetics and gene mutations influence survival in older patients with acute myeloid leukemia treated with azacitidine or conventional care. Leukemia. 2018;32 (12):2546-2557.

7. Döhner H, Estey E, Grimwade D, et al. Diagnosis and management of AML in adults: 2017 ELN recommendations from an international expert panel. Blood. 2017;129(4):424-447.

8. de Witte T, Hagemeijer A, Suciu S, et al. Value of allogeneic versus tautologous stem cell transplantation and chemotherapy in patients with myelodysplastic syndromes and secondary acute myeloid leukemia. Final results of a prospective randomized European intergroup trial. Haematological. 2010;95(10):1754-1761.

9. Lindsley RC, Mar BG, Mazzola E, et al. Acute myeloid leukemia ontogeny is defined by distinct somatic mutations. Blood. 2015;125(9):1367-1376.

10. Haferlach T, Nagata Y, Grossmann V, et al. Landscape of genetic lesions in 944 patients with myelodys-plastic syndromes. Leukemia. 2014;28(2):241-247.

11. Makishima H, Yoshizato T, Yoshida K, et al. Dynamics of clonal evolution in myelodysplastic syndromes. Nat Genet. 2017;49(2):204-212.

12. Vannucchi AM, Lasho TL, Guglielmelli P, et al. Mutations and prognosis in primary myelofibrosis. Leu-kemia. 2013;27(9):18611869.

13. Lasho TL, Mudireddy M, Fink CM, et al. Targeted next-generation sequencing in blast phase myelopro-liferative neoplasms. Blood Adv. 2018;2(4):370-380.

14. Papaemmanuil E, Gerstung M, Bullinger L, et al. Genomic classification and prognosis in acute myeloid leukemia. $\mathrm{N}$ Engl J Med. 2016;374(23):2209-2221.

15. Metzeler KH, Herold T, RothenbergThurley M, et al. Spectrum and prognostic relevance of driver gene mutations in acute myeloid leukemia. Blood. 2016;128(5):686698.

16. Dicker F, Haferlach C, Sundermann J, et al. Mutation analysis for RUNX1, MLL-PTD, FLT3-ITD, NPM1 and NRAS in 269 patients with MDS or secondary AML. Leukemia. 2010;24(8):1528-1532.

17. Bassan R, Intermesoli T, Masciulli A, et al. Randomized trial comparing standard vs sequential high-dose chemotherapy for inducing early CR in adult AML. Blood Adv. 2019;3(7):1103-1117.

18. Salmoiraghi S, Cavagna $R$, Zanghì $P$, et al. High throughput molecular characterizaion of normal karyo-type acute myeloid leukemia in the context of the prospective trial $02 / 06$ of the Northern Italy Leukemia Group (NILG). Cancers. 2020;12(8):2242.

19. Cheson BD, Bennett JM, Kopecky KJ, et al. Revised recommendations of the International Working Group for Diagnosis, Standardization of Response Criteria, Treatment Outcomes, and Reporting Standards for therapeutic trials in acute myeloid leukemia. J Chin Oncol.
2003;21(24):4642-4649.

20. Levine RL, Valk PJM. Next-generation sequencing in the diagnosis and minimal residual disease assessment of acute myeloid leukemia. Haematological. 2019; 104(5):868-871.

21. Papaemmanuil E, Gerstung M, Malcovati L, et al. Clinical and biological implications of driver mutations in myelodysplastic syndromes. Blood. 2013;122(22):3616-3627.

22. Ley TJ, Miller C, Ding L, et al. Genomic and epigenomic landscapes of adult de nov acute myeloid leukemia. N Engl J Med. 2013;368(22):2059-2074.

23. Cardin C, Pautas C, Fournier E, et al. Added prognostic value of secondary AML-like gene mutations in ELN intermediate-risk older AML: ALFA-1200 study results. Blood Adv. 2020;4(9):1942-1949.

24. Brierley CK, Steensma DP. Targeting spliceosome in the treatment of myelodysplastic syndromes and other myeloid neoplasms. Gur Hematol Malig Rep. 2016;11 (6):408-415.

25. Steensma DP, Maris MB, Yang J, et al. H3B8800-G0001-101: a first in human phase I study of a spliceosome modulator in patients with advanced myeloid malignancries. J Chin Oncol. 2017;35(Suppl 15): TPS7075.

26. Daigle SR, Olhava EJ, Therkelsen CA, et al. Selective killing of mixed lineage leukemia cells by a potent small-molecule DOT1L inhibitor. Cancer Cell. 2011;20(1):53-65.

27. Stein EM, DiNardo CD, Pollyea DA, et al. Enasidenib in mutant IDH2 relapsed or refractory acute myeloid leukemia. Blood. 2017;130(6):722-731.

28. Röllig C, Kramer M, Schliemann C, et al. Does time from diagnosis to treatment affect the prognosis of patients with newly diagnosed acute myeloid leukemia? Blood. 2020;136(7):823-830.

haematological | 2021; 106(10)

2587 